\title{
Decentralised Sliding Mode Control for Nonlinear Interconnected Systems in the Regular Form
}

\author{
Jianqiu $\mathrm{Mu}^{1}$, Xing-Gang $\operatorname{Yan}^{1}$, Sarah K. Spurgeon ${ }^{1}$ and Zehui Mao ${ }^{2}$
}

\begin{abstract}
In this paper, a decentralised control strategy based on sliding mode techniques is proposed for a class of nonlinear interconnected systems in regular form. All the isolated subsystems and interconnections are fully nonlinear. It is not required that the nominal isolated subsystems are either linearizable or partially linearizable. The uncertainties are nonlinear and bounded by nonlinear functions. Specifically, uncertainties in the input distribution and interconnections are considered. Under mild conditions, sliding mode controllers for each subsystem are designed by only employing local information. Sufficient conditions are developed under which information on the interconnections is employed for decentralised controller design to reduce conservatism. The bounds on the uncertainties have more general forms compared with previous work. A simulation example is used to demonstrate the effectiveness of the proposed method.
\end{abstract}

\section{INTRODUCTION}

In engineering, large scale systems are often modelled as a collection of subsystems with interconnections, e.g. multimachine power systems (e.g. see [18], [4]), power networks and energy systems (e.g. see [13]). Due to the complex dynamics caused by nonlinearity in the interconnections and subsystems, it is difficult to control such systems using classical methods. In reality, since such class of systems are usually distributed in different space, a centralised strategy can be difficult to motivate [20], [19] as the control for each subsystem requires all the information on the other subsystems and the control performance is highly dependent on efficient and reliable information exchange between subsystems. The reliability of information transfer among subsystems can be greatly affected by problems such as network failure or blockage of communication channels. Besides, time delay caused during the transfer process in the network may also reduce the system performance even if the communication channels are unimpeded. Therefore, the development of decentralised control strategies in which each subsystem is controlled independently is of interests. Since in decentralised strategy, the control of each subsystem only requires local information, which not only enhances system reliability but also reduces the costs of communication networks and their maintenance.

For nonlinear systems, it is well known that uncertainties or modelling errors may seriously affect control system per-

\footnotetext{
${ }^{1}$ Jianqiu Mu, Xing-Gang Yan and Sarah K. Spurgeon are with Instrumentation, Control and Embedded Systems Research Group, School of Engineering \& Digital Arts, University of Kent, CT2 7NT Canterbury, United Kingdom. Email: jm838@kent.ac.uk; x.yan@kent.ac.uk; s.k.spurgeon@kent.ac.uk

${ }^{2}$ Zehui Mao is with College of Automation Engineering, Nanjing University of Aeronautics and Astronautics, Nanjing, 210016, China. Email: zehuimao@nuaa.edu.cn
}

formance. In nonlinear interconnected systems, the effect of uncertainties on the whole system is even more challenging. To be specific, uncertainties experienced by a subsystem will affect not only its own performance but also the other subsystems' through the interactions between subsystems. Designing a decentralised control scheme to reject or reduce the effect of uncertainties in the interconnection terms is challenging. Sliding mode control has been recognised as a powerful approach in dealing with nonlinear systems with uncertainties [16] owing to its special structure and complete robustness against matched uncertainties [3], [16]. In [21], it is shown that the sliding mode approach can be used to deal with the systems in the presence of unmatched uncertainty. Methods proposed by Niu in [12] and [11] also show the strong robustness of sliding mode control for uncertain system. Therefore, many researchers have tried to develop a decentralised sliding mode control strategy for large scale system in the presence of uncertainties and interconnections. However, as the information available to the controllers of each subsystem is limited in a decentralised strategy, it is difficult to reject the uncertainties within the interconnections even if they are matched [17].

For robust decentralised controller design problems, many results have been obtained using various methods. In [14], [1], [6], [9], robust control strategy is used for the interconnected system. However, only matched uncertainties are considered and the bounds on the matched uncertainties are assumed to be linear or polynomial. In [21], mismatched uncertainties are considered with centralised dynamical feedback controllers which need more resources to exchange information between subsystems. In [15], a decentralised state feedback controller is proposed for systems with a class of constraints called integral quadratic constraints to limit the structure of the original system. In some cases, adaptive techniques are applied to estimate an upper bound on the mismatched uncertainty, and this is used to counteract the effects of uncertainty [2]. This approach is powerful for the case where the uncertainty satisfies a linear growth condition. In terms of mismatched uncertainties, it is inevitable to impose some limitations in order to achieve asymptotic stability. After transforming the system into a special triangular structure, the uncertainties of the system in [7] have more general forms when compared with previous work. For uncertainties in the input distribution, the existing research is limited. In [12] and [11], sliding mode control design is proposed for systems with uncertainties in the control matrix. However, the uncertainties in [12], [11] are assumed to have a particular structure, for example, the uncertainty is required 
to lie in the range of the control input distribution matrix. In most previous work, the nominal part of the system is usually assumed to be linear, which limits the application of the obtained results. In [8], a decentralised quantitative feedback control is proposed for a class of large-scale systems in the presence of uncertainties in the state-space matrices, and the work has also been implemented on a SCARA robot system. However, both the nominal part of the system and the interconnection between subsystem are assumed to be linear.

In this paper, a nonlinear decentralised control strategy for a class of nonlinear interconnected systems is proposed based on a sliding mode control paradigm. Compared with previous work in [10], the interconnected system is assumed to be fully nonlinear with unknown interconnections and uncertainties in the input distribution term. Moreover, the uncertainties are assumed to be bounded by known functions which are employed in the control design to counteract the effects of the uncertainties on the controlled interconnected system. The bounds on the uncertainties take more general forms when compared with existing work. A set of sufficient conditions is developed such that the corresponding sliding motion is asymptotically stable when the system is restricted to the designed sliding surface. Then, a decentralised sliding mode control is designed to drive the interconnected system to the sliding surface in the presence of uncertainties. It is also shown that if the uncertainties/interconnections possess a superposition property, a decentralised control scheme may be designed to counteract the effect of the uncertainty. A numerical example is presented with simulation results to show the effectiveness of the approach proposed.

\section{SySTEM DESCRIPTION}

Consider a class of nonlinear large-scale interconnected systems composed of $N$ subsystems where the $i$-th subsystem can be transformed or described by

$$
\begin{aligned}
\dot{x}_{i}^{a} & =f_{i}^{a}\left(t, x_{i}^{a}, x_{i}^{b}\right)+\psi_{i}^{a}(t, x) \\
\dot{x}_{i}^{b} & =f_{i}^{b}\left(t, x_{i}\right)+\left(g_{i}\left(t, x_{i}\right)+\phi_{i}\left(t, x_{i}\right)\right) u_{i}+\psi_{i}^{b}(t, x)
\end{aligned}
$$

where the state variables of the $i$-th subsystem are represented by $x_{i}:=\operatorname{col}\left(x_{i}^{a}, x_{i}^{b}\right) \subset \Omega_{i} \in \mathcal{R}^{n_{i}}$ where $x_{i}^{a} \in$ $\mathcal{R}^{n_{i}-m_{i}}, x_{i}^{b} \in \mathcal{R}^{m_{i}}$ and $x=\operatorname{col}\left(x_{1}, x_{2}, \ldots, x_{N}\right) . u_{i} \in \mathcal{R}^{m_{i}}$ denote inputs of the $i$-th subsystem respectively for $i=$ $1,2, \ldots, N$. The function $f_{i}^{a}(\cdot), f_{i}^{b}(\cdot)$ with $f_{i}^{a}(t, 0,0)=0$ and $f_{i}^{b}(t, 0)=0$ and the function matrix $g_{i}(\cdot)$ are continuous with appropriate dimensions. Uncertainty in the input distribution is denoted by $\phi_{i}\left(t, x_{i}\right)$. The nonlinear functions $\psi_{i}^{a}(t, x) \in \mathcal{R}^{n_{i}-m_{i}}$ and $\psi_{i}^{b}(t, x) \in \mathcal{R}^{m_{i}}$ represents the uncertain interconnection. It is assumed that all the nonlinear functions are sufficiently smooth such that the unforced system has a unique continuous solution.

In this paper, the focus is to design a decentralised control scheme to stabilise system (1)-(2) under the assumptions that the isolated nominal system has the desired performance. The following basic assumptions are imposed on the uncertainties of the system (1)-(2).
Assumption 1. There exist known continuous functions $\delta_{i j}^{a}(\cdot), \delta_{i}^{b}(\cdot)$ and $\rho_{i}(\cdot)$ in $\mathcal{R}^{+}$with $\delta_{i j}^{a}(t, 0)=0$ such that

$$
\begin{aligned}
\text { (i) }\left\|\psi_{i}^{a}(t, x)\right\| & \leq \sum_{j=1}^{n} \delta_{i j}^{a}\left(\left\|x_{j}\right\|\right) \\
\text { (ii) }\left\|\psi_{i}^{b}(t, x)\right\| & \leq \delta_{i}^{b}(t, x) \\
\text { (iii) }\left\|\phi_{i}\left(t, x_{i}\right)\right\| & \leq \rho_{i}\left(t, x_{i}\right)
\end{aligned}
$$

for all $t \in \mathcal{R}^{+}, x_{i} \in \Omega_{i}$.

Assumption 2. The function matrix $g_{i}\left(t, x_{i}\right)$ is nonsingular for any $\left(t, x_{i}\right) \in \mathcal{R}^{+} \times \Omega_{i}$, and the uncertainty $\phi_{i}\left(t, x_{i}\right)$ in system (2) satisfies

$$
g_{i}^{-1}\left(t, x_{i}\right) \phi_{i}\left(t, x_{i}\right)+\phi_{i}^{\tau}\left(t, x_{i}\right)\left(g_{i}^{-1}\left(t, x_{i}\right)\right)^{\tau} \geq 0
$$

for all $t \in \mathcal{R}^{+}, x_{i} \in \Omega_{i}$.

Remark 1. Assumption 2 is made on the uncertainties in the input distribution term. It will be shown that a class of uncertainties in the input distribution can be rejected by designing an appropriate control. Comparing with existing work, e.g. see [12], [11], [8], the uncertainties in the input distribution are nonlinear instead of linear. It is emphasised that the uncertainties $\phi_{i}(\cdot)$ are not required to be matched, and only $g_{i}^{b}(\cdot)$ is required to be nonsingular for $t \in \mathcal{R}^{+}$, $x_{i} \in \Omega_{i}$.

Since $\phi_{i}\left(t, x_{i}\right)$ are the uncertainties in the input distribution, their effects are closely related to the control signal $u_{i}$ for $i=1,2, \ldots, N$. This can be seen from the terms $\phi_{i}\left(t, x_{i}\right) u_{i}$ in system (2). Therefore, the uncertainties existing in the input distribution make the control design much more difficult. This paper will present an approach to deal with nonlinear uncertainties in the input distribution when the input distribution is nonlinear.

\section{Stability analysis of the Sliding Mode}

Choose the local sliding surface for the $i$ th subsystem of the large-scale interconnected system (1)-(2) as follows:

$$
\sigma_{i}\left(x_{i}\right) \equiv: x_{i}^{b}=0, \quad i=1,2, \ldots, N
$$

Then, the composite sliding surface for the interconnected system (1)-(2) is chosen as

$$
\sigma(x)=0
$$

where $\sigma(x) \equiv: \operatorname{col}\left(x_{1}^{b}, x_{2}^{b}, \ldots, x_{N}^{b}\right)$. During sliding motion, $x_{i}^{b}=0$ for $i=1,2, \ldots, N$. Then, the sliding mode dynamics for the system (1)-(2) associated with the designed sliding surface $(8)$ can be described by

$$
\dot{x}_{i}^{a}=f_{i}^{a}\left(t, x_{i}^{a}, 0\right)+\psi_{i}^{s}\left(t, x_{1}^{a}, x_{2}^{a}, \ldots, x_{N}^{a}\right)
$$

where

$$
\psi_{i}^{s}\left(t, x_{1}^{a}, x_{2}^{a}, \ldots, x_{N}^{a}\right):=\left.\psi_{i}^{a}(t, x)\right|_{\left(x_{1}^{b}, x_{2}^{b}, \ldots, x_{N}^{b}\right)=0}
$$

with $\psi_{i}^{a}(t, x)$ defined in (1). 
Lemma 1. For terms $\psi_{i}^{s}\left(t, x_{1}^{a}, x_{2}^{a}, \ldots, x_{N}^{a}\right)$ in system (9), if inequality (3) in Assumption 1 holds, then

$$
\left\|\psi_{i}^{s}\left(t, x_{1}^{a}, x_{2}^{a}, \ldots, x_{N}^{a}\right)\right\| \leq \sum_{j=1}^{N} \delta_{i j}^{a}\left(\left\|x_{j}^{a}\right\|\right)
$$

where $x^{a}=\operatorname{col}\left(x_{1}^{a}, x_{2}^{a}, \ldots, x_{N}^{a}\right)$ and $\delta_{i j}(\cdot)$ satisfy (3).

Proof. From the definition of $\psi_{i}^{s}(\cdot)$ in (10), it follows that

$$
\psi_{i}^{s}\left(t, x_{1}^{a}, x_{2}^{a}, \ldots, x_{N}^{a}\right)=\psi_{i}^{a}\left(t, x_{1}^{a}, 0, x_{2}^{a}, 0, \ldots, x_{N}^{a}, 0\right)
$$

From (3) in Assumption 1,

$$
\left\|\psi_{i}^{a}(t, x)\right\| \leq \sum_{j=1}^{N} \delta_{i j}^{a}\left(\left\|x_{j}\right\|\right)
$$

From (12) and (13), it follows that

$$
\begin{aligned}
\left\|\psi_{i}^{s}\left(t, x_{1}^{a}, x_{2}^{a}, \ldots, x_{N}^{a}\right)\right\| & =\left\|\psi_{i}^{a}\left(t, x_{1}^{a}, 0, x_{2}^{a}, 0, \ldots, x_{N}^{a}, 0\right)\right\| \\
& \leq \sum_{j=1}^{N} \delta_{i j}^{a}\left(\left\|x_{j}^{a}\right\|\right)
\end{aligned}
$$

Hence the result follows.

Assumption 3. There exist continuous $C^{1}$ function $V_{i}$ : $\mathcal{R}^{+} \times \mathcal{R}^{n_{i}-m_{i}} \rightarrow \mathcal{R}^{+}$and functions $\varsigma_{i 1}(\cdot), \varsigma_{i 2}(\cdot), \varsigma_{i 3}(\cdot)$ and $\varsigma_{i 4}(\cdot)$ of class $\mathcal{K}$ such that for all $x_{i} \in \Omega_{i}$ and $t \in \mathcal{R}^{+}$

(i) $\varsigma_{i 1}\left(\left\|x_{i}^{a}\right\|\right) \leq V_{i}\left(t, x_{i}^{a}\right) \leq \varsigma_{i 2}\left(\left\|x_{i}^{a}\right\|\right)$

(ii) $\frac{\partial V_{i}\left(t, x_{i}^{a}\right)}{\partial t}+\frac{\partial V_{i}\left(t, x_{i}^{a}\right)}{\partial x_{i}^{a}} f_{i}^{a}\left(t, x_{i}^{a}, 0\right)$ $\leq-\varsigma_{i 3}^{2}\left(\left\|x_{i}^{a}\right\|\right)$

(iii) $\left\|\frac{\partial V_{i}\left(t, x_{i}^{a}\right)}{\partial x_{i}^{a}}\right\| \leq \varsigma_{i 4}\left(\left\|x_{i}^{a}\right\|\right)$

where

$$
\frac{\partial V_{i}\left(t, x_{i}^{a}\right)}{\partial x_{i}^{a}}=\left(\frac{\partial V_{i}\left(t, x_{i}^{a}\right)}{\partial x_{1}^{a}}, \frac{\partial V_{i}\left(t, x_{i}^{a}\right)}{\partial x_{2}^{a}} \ldots \frac{\partial V_{i}\left(t, x_{i}^{a}\right)}{\partial x_{n}^{a}}\right)
$$

Theorem 1. Under assumptions 1-3, the sliding mode (9) of the system (1)-(2) associated with the sliding surface in (8) is asymptotically stable if there exists a domain $\Omega_{x^{a}}$ of the origin in $x^{a} \in \mathcal{R}^{\sum_{i}^{N}\left(n_{i}-m_{i}\right)}$ such that

$$
M(t, x)^{T}+M(t, x)>0
$$

in domain $\Omega_{x^{a}} \backslash\{0\}$ with $M(t, x)=\left(m_{i j}\left(t, x_{i}, x_{j}\right)\right)_{N \times N}$ and for $i, j=1,2, \ldots, N$

$m_{i j}\left(t, x_{i}, x_{j}\right)= \begin{cases}\mu_{i 3}\left(\left\|x_{i}^{a}\right\|\right)-\mu_{i 4}\left(\left\|x_{i}^{a}\right\|\right) \gamma_{i i}\left(\left\|x_{i}^{a}\right\|\right), & i=j \\ -\mu_{i 4}\left(\left\|x_{i}^{a}\right\|\right) \gamma_{i j}\left(\left\|x_{j}^{a}\right\|\right), & i \neq j\end{cases}$

where $\mu_{i 3}(\cdot), \mu_{i 4}(\cdot)$ and $\gamma_{i j}(\cdot)$ are defined respectively by

$$
\begin{aligned}
& \mu_{i 3}(x)=\int_{0}^{1} \frac{\partial \varsigma_{i 3}(x \tau)}{\partial \tau} \mathrm{d} \tau \\
& \mu_{i 4}(x)=\int_{0}^{1} \frac{\partial \varsigma_{i 4}(x \tau)}{\partial \tau} \mathrm{d} \tau \\
& \gamma_{i j}(x)=\int_{0}^{1} \frac{\partial \delta_{i j}^{a}(x \tau)}{\partial \tau} \mathrm{d} \tau
\end{aligned}
$$

Proof. From (15)-(17), it can be observed that

$$
\begin{aligned}
& \varsigma_{i 3}\left(\left\|x_{i}^{a}\right\|\right)=\mu_{i 3}\left(\left\|x_{i}^{a}\right\|\right)\left\|x_{i}^{a}\right\| \\
& \varsigma_{i 4}\left(\left\|x_{i}^{a}\right\|\right)=\mu_{i 4}\left(\left\|x_{i}^{a}\right\|\right)\left\|x_{i}^{a}\right\| \\
& \delta_{i j}^{a}\left(\left\|x_{i}^{a}\right\|\right)=\gamma_{i j}\left(\left\|x_{i}^{a}\right\|\right)\left\|x_{i}^{a}\right\|
\end{aligned}
$$

From the analysis above, it is seen that the system (9) represents the sliding mode dynamics of the system (1)-(2) corresponding to the sliding surface (8).

For system (9), consider the Lyapunov function candidate

$$
V\left(t, x_{i}^{a}\right)=\sum_{i=1}^{N} V_{i}\left(t, x_{i}^{a}\right)
$$

where $V_{i}\left(t, x_{i}^{a}\right)$ is given by Assumption 3. Then, the time derivative of $V\left(t, x_{i}^{a}\right)$ along equation (9) is given by

$$
\begin{aligned}
\dot{V}= & \sum_{i=1}^{N}\left\{\frac{\partial V_{i}\left(t, x_{i}^{a}\right)}{\partial t}+\frac{\partial V_{i}\left(t, x_{i}^{a}\right)}{\partial x_{i}^{a}} f_{i}^{a}\left(t, x_{i}^{a}, 0\right)\right. \\
& \left.+\frac{\partial V_{i}\left(t, x_{i}^{a}\right)}{\partial x_{i}^{a}} \psi_{i}^{a}(t, x)\right\} \\
\leq & \sum_{i=1}^{N}\left\{-\varsigma_{i 3}^{2}\left(\left\|x_{i}^{a}\right\|\right)+\varsigma_{i 4}\left(\left\|x_{i}^{a}\right\|\right) \sum_{j=1}^{N} \delta_{i j}\left(\left\|x_{j}^{a}\right\|\right)\right\} \\
= & -\sum_{i=1}^{N} \mu_{i 3}^{2}\left(\left\|x_{i}^{a}\right\|\right)\left\|x_{i}^{a}\right\|^{2} \\
& +\sum_{i=1}^{N} \sum_{j=1}^{N} \mu_{i 4}\left(\left\|x_{i}^{a}\right\|\right) \gamma_{i j}\left(\left\|x_{j}^{a}\right\|\right)\left\|x_{i}^{a}\right\|\left\|x_{j}^{a}\right\| \\
= & -\frac{1}{2}\left(\left\|x_{1}^{a}\right\|,\left\|x_{2}^{a}\right\|, \ldots,\left\|x_{N}^{a}\right\|\right)\left(M^{T}+M\right)\left(\begin{array}{c}
\left\|x_{1}^{a}\right\| \\
\left\|x_{2}^{a}\right\| \\
\vdots \\
\left\|x_{N}^{a}\right\|
\end{array}\right)
\end{aligned}
$$

Since the matrix function $M^{T}+M$ in $\Omega_{x^{a}} \backslash\{0\}$ is positive definite, therefore, it follows that $V$ is a negative definite function in Domain $\Omega_{x^{a}}$. Hence, the results follow.

\section{Decentralised Control Design}

For the nonlinear interconnected system (1)-(2), the corresponding reachability condition is described by (e.g. see [5], [17])

$$
\sum_{i=1}^{N} \frac{\sigma_{i}^{\tau}\left(x_{i}\right) \dot{\sigma}_{i}\left(x_{i}\right)}{\left\|\sigma_{i}\left(x_{i}\right)\right\|}<0
$$

where $\sigma_{i}\left(x_{i}\right)$ is defined by (7). In order to reduce the effects of the unknown interconnection $\psi_{i}^{b}(\cdot)$, consider the expression

$$
\delta_{i}^{b}(t, x)=\sum_{j=1}^{N} \eta_{i j}\left(t, x_{j}\right)+\nu_{i}(t, x)
$$

where $\delta_{i}^{b}$ is defined in (4) and $\nu_{i}(t, x)$ represents all the coupling terms which cannot be included in the term $\sum_{j=1}^{N} \eta_{i j}\left(t, x_{j}\right)$ Consider the decentralised control

$$
u_{i}=u_{i}^{a}+u_{i}^{b}
$$


where

$$
\begin{aligned}
u_{i}^{a}= & -g_{i}^{-1}\left(t, x_{i}\right) f_{i}^{b}\left(t, x_{i}\right) \\
& -g_{i}^{-1}\left(t, x_{i}\right) \operatorname{sgn}\left(x_{i}^{b}\right)\left\{\sum_{j=1}^{N} \eta_{j i}\left(t, x_{i}\right)+\zeta_{i}\left(t, x_{i}\right)\right\} \\
u_{i}^{b}= & -g_{i}^{-1}\left(t, x_{i}\right) \operatorname{sgn}\left(x_{i}^{b}\right)\left\|u_{i}^{a}\right\| \rho_{i}\left(t, x_{i}\right)
\end{aligned}
$$

where the $\rho_{i}\left(t, x_{i}\right)$ are defined in Assumption 1, and $\eta_{j i}\left(t, x_{i}\right)$ satisfy (23). The term $\zeta_{i}\left(t, x_{i}\right)$ is a reachability function which can be considered as a design parameter to be defined.

Theorem 2. Consider the nonlinear interconnected system (1)-(2). Under Assumptions 1-3, the closed-loop system (1)(2) with the decentralised control (24) is converged to the composite sliding surface (8) and maintain a sliding motion on it thereafter if in the considered domain $\Omega=\Omega_{1} \times \Omega_{2} \cdots \times$ $\Omega_{N}$, the functions $\zeta_{i}\left(t, x_{i}\right)$ in (25) satisfy

$$
\sum_{i=1}^{N} \zeta_{i}\left(t, x_{i}\right)>\sum_{i=1}^{N} \nu_{i}(t, x)
$$

in $\Omega$ for all $t>0$ with $\nu_{i}(t, x)$ defined in (23).

Proof. From (7), for $i=1,2, \ldots, N$

$$
\begin{aligned}
\dot{\sigma}_{i}\left(x_{i}\right)= & f_{i}^{b}\left(t, x_{i}\right)+\left(g_{i}^{b}\left(t, x_{i}\right)+\phi_{i}\left(t, x_{i}\right)\right)\left(u_{i}^{a}+u_{i}^{b}\right) \\
& +\psi_{i}^{b}(t, x)
\end{aligned}
$$

Substituting (25)-(26) into (28),

$$
\begin{aligned}
\sum_{i=1}^{N} & \frac{\sigma_{i}^{\tau}\left(x_{i}\right) \dot{\sigma}_{i}\left(x_{i}\right)}{\left\|\sigma_{i}\left(x_{i}\right)\right\|} \\
= & \sum_{i=1}^{N}\left\{\frac{\left(x_{i}^{b}\right)^{\tau}}{\left\|x_{i}^{b}\right\|}\left\{\delta_{i}^{b}(t, x)+\phi_{i}\left(t, x_{i}\right) u_{i}^{a}\right\}\right. \\
& -\frac{\left(x_{i}^{b}\right)^{\tau}}{\left\|x_{i}^{b}\right\|} \phi_{i}\left(t, x_{i}\right)\left(g_{i}^{b}\left(t, x_{i}\right)\right)^{-1}\left\|u_{i}^{a}\right\| \rho_{i}\left(t, x_{i}\right) \operatorname{sgn}\left(x_{i}^{b}\right) \\
& \left.-\sum_{j=1}^{N} \mu_{j i}\left(t, x_{i}\right)-\zeta_{i}\left(t, x_{i}\right)-\left\|u_{i}^{a}\right\| \rho_{i}\left(t, x_{i}\right)\right\} \\
\leq & \sum_{i=1}^{N}\left\|\phi_{i}\left(t, x_{i}\right) u_{i}^{a}\right\|+\sum_{i=1}^{N}\left\|\delta_{i}^{b}(t, x)\right\| \\
& -\sum_{i=1}^{N} \frac{\left(x_{i}^{b}\right)^{\tau}}{\left\|x_{i}^{b}\right\|} \phi_{i}\left(t, x_{i}\right)\left(g_{i}^{b}\left(t, x_{i}\right)\right)^{-1}\left\|u_{i}^{a}\right\| \rho_{i}\left(t, x_{i}\right) \operatorname{sgn}\left(x_{i}^{b}\right) \\
& -\sum_{i=1}^{N}\left\|u_{i}^{a}\right\| \rho_{i}\left(t, x_{i}\right)-\sum_{i=1}^{N} \sum_{j=1}^{N} \mu_{j i}\left(t, x_{i}\right)-\sum_{i=1}^{N} \zeta_{i}\left(t, x_{i}\right)
\end{aligned}
$$

From Assumption 1,

$$
\begin{aligned}
\sum_{i=1}^{N}\left\|\delta_{i}^{b}\left(t, T^{-1} x\right)\right\| & \leq \sum_{i=1}^{N} \sum_{j=1}^{N} \mu_{i j}\left(t, x_{j}\right)+\sum_{i=1}^{N} \nu_{i}(t, x) \\
& =\sum_{i=1}^{N} \sum_{j=1}^{N} \mu_{j i}\left(t, x_{i}\right)+\sum_{i=1}^{N} \nu_{i}(t, x)
\end{aligned}
$$

$$
\begin{aligned}
\left\|\phi_{i}\left(t, x_{i}\right) u_{i}^{a}\right\| & \leq\left\|\phi_{i}\left(t, x_{i}\right)\right\|\left\|u_{i}^{a}\right\| \\
& \leq\left\|u_{i}^{a}\right\| \rho_{i}\left(t, x_{i}\right)
\end{aligned}
$$

and from Assumption 2,

$$
\begin{aligned}
& \frac{\left(x_{i}^{b}\right)^{\tau}}{\left\|x_{i}^{b}\right\|} \phi_{i}\left(t, x_{i}\right) g_{i}^{-1}\left(t, x_{i}\right)\left\|u_{i}^{a}\right\| \rho_{i}\left(t, x_{i}\right) \operatorname{sgn}\left(x_{i}^{b}\right) \\
= & \frac{\left(x_{i}^{b}\right)^{\tau}}{\left\|x_{i}^{b}\right\|}\left\|u_{i}^{a}\right\| \rho_{i}\left(t, x_{i}\right) \\
& \cdot \frac{\left(\phi_{i}\left(t, x_{i}\right) g_{i}^{-1}\left(t, x_{i}\right)+\left(g_{i}^{-1}\left(t, x_{i}\right)\right)^{\tau} \phi_{i}^{\tau}\left(t, x_{i}\right)\right)}{2} \\
& \cdot \operatorname{sgn}\left(x_{i}^{b}\right) \geq 0
\end{aligned}
$$

Substituting inequalities (30), (31) and (32) into (29),

$$
\sum_{i=1}^{N} \frac{\sigma_{i}^{\tau} \dot{\sigma}_{i}}{\left\|\sigma_{i}\right\|}<-\sum_{i=1}^{N} \zeta_{i}\left(t, x_{i}\right)+\sum_{i=1}^{N} \nu_{i}(t, x)<0
$$

Then the reachability condition (22) is satisfied. Hence, the result follows.

From sliding mode control theory, Theorems 1 and 2 together guarantee that the system (1)-(2) is stabilized by the designed decentralised control (24) with $u_{i}^{a}$ and $u_{i}^{b}$ defined in (25) and (26).

\section{NUMERICAL SIMULATION}

Consider the following nonlinear interconnected systems composed of the three subsystems described by

$$
\begin{aligned}
& \dot{x}_{1}^{a}=\underbrace{\frac{x_{11} x_{12}-4 x_{11} \cos \left(x_{13}\right)}{\sqrt{1+x_{12}^{2}+x_{13}^{2}}}}_{f_{1}^{a}\left(t, x_{1}^{a}, x_{1}^{b}\right)}+\psi_{1}^{a}(t, x) \\
& \dot{x}_{1}^{b}=\underbrace{\left[\begin{array}{c}
1.6 x_{11} x_{12} \\
\frac{1.5 x_{12}}{x_{13}^{2}+1}
\end{array}\right]}_{f_{1}^{b}\left(t, x_{1}\right)}+\psi_{1}^{b}(t, x) \\
& +(\underbrace{\left[\begin{array}{cc}
1 & x_{12} \\
0 & 1
\end{array}\right]}_{g_{1}\left(t, x_{1}\right)}+\phi_{1}\left(t, x_{1}\right)) u_{1} \\
& \dot{x}_{2}^{a}=\underbrace{\frac{x_{21} x_{22}-6.25 x_{21} \cos \left(x_{22}\right)}{1+0.1 x_{22}^{2}}}_{f_{2}^{a}\left(t, x_{2}^{a}, x_{2}^{b}\right)}+\psi_{2}^{a}(t, x) \\
& \dot{x}_{2}^{b}=\underbrace{1.3 x_{21} x_{22}}_{f_{2}^{b}\left(t, x_{2}^{a}, x_{2}^{b}\right)}+\psi_{2}^{b}(t, x) \\
& +(\underbrace{\sin ^{2}\left(x_{22}\right)+1}_{g_{2}\left(t, x_{2}\right)}+\phi_{2}\left(t, x_{2}\right)) u_{2}
\end{aligned}
$$




$$
\begin{aligned}
\dot{x}_{3}^{a}= & \underbrace{\left[\begin{array}{c}
x_{32}-3.61 x_{32} \cos \left(x_{33}\right) \\
-x_{31}+\left(x_{33}^{2}-4\right) x_{32}
\end{array}\right]}_{f_{3}^{a}\left(t, x_{3}^{a}, x_{3}^{b}\right)}+\psi_{3}^{a}(t, x) \\
\dot{x}_{3}^{b}= & \underbrace{\frac{1.6 x_{32}}{x_{33}^{2}+1}+\psi_{3}^{b}(t, x)}_{f_{3}^{b}\left(t, x_{3}^{a}, x_{3}^{b}\right)} \\
& +(\underbrace{\cos ^{2}\left(x_{31} x_{33}\right)+0.8}_{g_{3}\left(t, x_{3}\right)}+\phi_{3}\left(t, x_{3}\right)) u_{3}
\end{aligned}
$$

where $x_{11}:=x_{1}^{a}, \operatorname{col}\left(x_{12}, x_{13}\right):=x_{2}^{b}, x_{21}:=x_{1}^{a}, x_{22}:=x_{2}^{b}$, $\operatorname{col}\left(x_{31}, x_{32}\right):=x_{1}^{a}$ and $x_{33}:=x_{2}^{b}$. Assume the uncertainties satisfy

$$
\begin{aligned}
& \left\|\phi_{1}\left(t, x_{1}\right)\right\| \leq 0.8\left|x_{12}\right|+0.7 \\
& \left\|\phi_{2}\left(t, x_{2}\right)\right\| \leq 0.3\left|x_{21} x_{22}\right| \\
& \left\|\phi_{3}\left(t, x_{3}\right)\right\| \leq 0.5\left|x_{31} \cos \left(x_{32}\right)\right|+0.2 \\
& \left\|\psi_{1}^{a}(t, x)\right\| \leq \underbrace{0.4 \frac{\left|x_{11} \sin \left(x_{11}\right)\right|}{\sin \left(x_{12}\right)^{2}+1}}_{\delta_{11}^{a}\left(\left\|x_{1}\right\|\right)} \\
& +\underbrace{0.3\left|x_{21}+x_{22}\right| \cos ^{2}\left(x_{21}\right)}_{\delta_{12}^{a}\left(\left\|x_{2}\right\|\right)}+\underbrace{\frac{0.2\left|x_{31}\right|}{1+x_{32}^{2}}}_{\delta_{13}^{a}\left(\left\|x_{3}\right\|\right)} \\
& \left\|\psi_{2}^{a}(t, x)\right\| \leq \underbrace{0.7\left\|x_{1}\right\|}_{\delta_{21}^{a}\left(\left\|x_{1}\right\|\right)}+\underbrace{\left|x_{21} \cos \left(x_{21}\right)\right|}_{\delta_{22}^{a}\left(\left\|x_{2}\right\|\right)}+\underbrace{0.1\left\|x_{3}\right\|}_{\delta_{23}^{a}\left(\left\|x_{3}\right\|\right)} \\
& \left\|\psi_{3}^{a}(t, x)\right\| \leq \underbrace{1.2\left|x_{11} \sin \left(x_{11}\right)\right|}_{\delta_{31}^{a}\left(\left\|x_{1}\right\|\right)}+\underbrace{0.8\left|x_{21}+x_{22}\right|}_{\delta_{32}^{a}\left(\left\|x_{2}\right\|\right)} \\
& +\underbrace{\left|x_{31} \cos \left(x_{32} x_{33}\right)\right|}_{\delta_{33}^{a}\left(\left\|x_{3}\right\|\right)} \\
& \left\|\psi_{1}^{b}(t, x)\right\| \leq \underbrace{0.1\|x\|}_{\nu_{1}(t, x)} \\
& \left\|\psi_{2}^{b}(t, x)\right\| \leq \underbrace{0.4\left\|x_{1}\right\|}_{\eta_{21}\left(t, x_{1}\right)}+\underbrace{\frac{0.2\left\|x_{2}\right\|}{\left\|x_{3}\right\|+1}}_{\nu_{2}(t, x)} \\
& \left\|\psi_{3}^{b}(t, x)\right\| \leq \underbrace{0.4\left\|x_{3}\right\|}_{\eta_{33}\left(t, x_{3}\right)}+\underbrace{0.1\left\|x_{1}\right\|\left\|x_{2}\right\|}_{\nu_{3}(t, x)}
\end{aligned}
$$

Now define the sliding function as

$$
\sigma\left(x_{i}\right)=x_{i}^{b} \quad i=1,2,3
$$

Then, from Lemma 1, when the sliding motion takes place,

$$
\begin{aligned}
\left\|\psi_{1}^{a}\left(t, x_{11}, x_{21}, x_{31}\right)\right\| \leq & \underbrace{0.4\left|x_{11} \sin \left(x_{11}\right)\right|}_{\delta_{11}^{a}\left(\left\|x_{1}^{a}\right\|\right)} \\
& +\underbrace{0.3\left|x_{21}\right| \cos ^{2}\left(x_{21}\right)}_{\delta_{12}^{a}\left(\left\|x_{2}^{a}\right\|\right)}+\underbrace{0.2\left|x_{31}\right|}_{\delta_{13}^{a}\left(\left\|x_{3}^{a}\right\|\right)}
\end{aligned}
$$

$$
\begin{aligned}
\left\|\psi_{2}^{a}\left(t, x_{11}, x_{21}, x_{31}\right)\right\| \leq & 0 \underbrace{0.7\left|x_{11}\right|}_{\delta_{21}^{a}\left(\left\|x_{1}^{a}\right\|\right)}+\underbrace{\left|x_{21} \cos \left(x_{21}\right)\right|}_{\delta_{22}^{a}\left(\left\|x_{2}^{a}\right\|\right)} \\
& +\underbrace{0.1\left|x_{31}\right|}_{\delta_{23}^{a}\left(\left\|x_{3}^{a}\right\|\right)} \\
\left\|\psi_{3}^{a}\left(t, x_{11}, x_{21}, x_{31}\right)\right\| \leq & \underbrace{1.2\left|x_{11} \sin \left(x_{11}\right)\right|}_{\delta_{31}^{a}\left(\left\|x_{1}^{a}\right\|\right)}+\underbrace{0.8\left|x_{21}\right|}_{\delta_{32}^{a}\left(\left\|x_{2}^{a}\right\|\right)} \\
& +\underbrace{\left|x_{31}\right|}_{\delta_{33}^{a}\left(\left\|x_{3}^{a}\right\|\right)}
\end{aligned}
$$

Choosing the Lyapunov function candidate

$$
V_{i}=\frac{1}{2}\left(x_{i}^{a}\right)^{T} x_{i}^{a}, \quad i=1,2,3
$$

Then,

$$
\underbrace{0.4\left\|x_{i}^{a}\right\|^{2}}_{\varsigma_{i 1}} \leq V_{i}\left(t, x_{i}^{a}\right) \leq 0.6 \underbrace{\left\|x_{i}^{a}\right\|^{2}}_{\varsigma_{i 2}}
$$

Define $\varsigma_{i 3}(\cdot)$ for $i=1,2,3$ as

$$
\varsigma_{13}(r)=2 r, \quad \varsigma_{23}(r)=2.5 r, \quad \varsigma_{33}(r)=1.9 r
$$

and $\varsigma_{i 4}(\cdot)$ as

$$
\varsigma_{i 4}(r)=r, \quad i=1,2,3
$$

Then, through direct computation, it is straightforward to verify that the conditions of Theorem 1 are satisfied. Thus the designed sliding mode is asymptotically stable.

From (25) and (26), the controller $u_{i}$ for $i=1,2,3$ are well defined with $\zeta_{1}=0.2\left\|x_{1}\right\|, \zeta_{2}=0.4\left\|x_{2}\right\|$ and $\zeta_{3}=$ $0.1\left\|x_{3}\right\|$ which guarantee the condition (27) in Theorem 2 is satisfied for $x_{i} \in \mathcal{R}^{3}, i=1,2,3$. Therefore system (34)-(39) can be stabilised by the designed control.

The time response of the system states is shown in Fig.1, and the time response of the control signal is shown in Fig. 2 . The simulation results show that the proposed approach is effective. It should be noted that in the simulation a boundary layer is used to reduce the chattering of the controller.

Remark 2. To illustrate the results, the uncertainties in the input distribution are chosen to satisfy (6) in Assumption 2, e.g. the term $\phi_{1}\left(t, x_{1}\right)$ is described by the form

$$
\phi_{1}\left(t, x_{1}\right)=\left[\begin{array}{cc}
\alpha-\beta & \alpha x_{12} \\
-\beta x_{12} & c
\end{array}\right]
$$

where $\alpha, \beta$ and $c$ are unknown parameters with $0 \leq \beta<$ $\alpha<0.8$ and $|c|<1$.

\section{CONCLUSION}

This paper has proposed a robust decentralised control design approach for a class of nonlinear systems with uncertainties in the input distribution and in the interconnection. The bounds on the uncertainties are assumed to be known functions which have been used to enhance robustness against the uncertainties. A sliding mode control is designed to guarantee reachability. The developed results can be applied to all interconnected systems which can be transfered to the regular form in (1)-(2). A numerical example is given to show how to use sliding mode techniques to stabilise a system with uncertainties in the input distributions. Simulations have been presented to demonstrate the effectiveness of this approach. 


\section{REFERENCES}
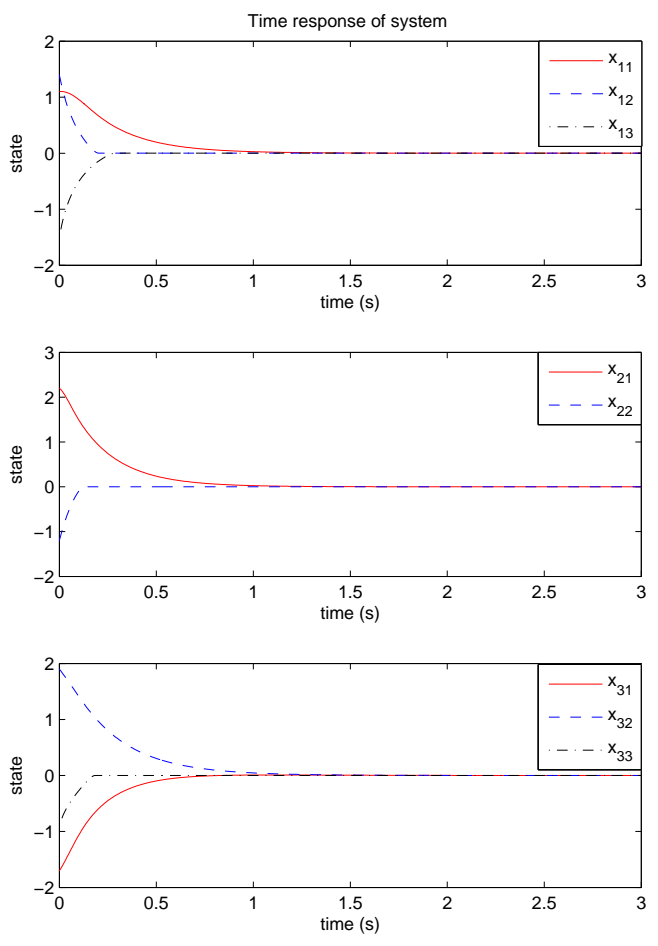

Fig. 1. Time response of state variables
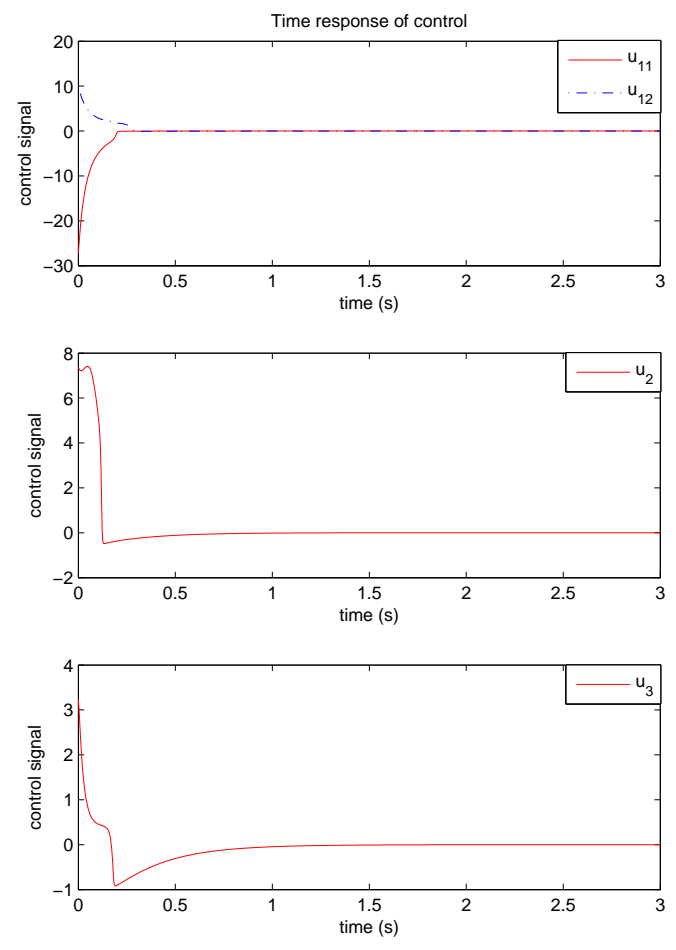

[1] Cheng-Fa Cheng. Disturbances attenuation for interconnected systems by decentralized control. International journal of control, 66(2):213224, 1997.

[2] Chih-Chiang Cheng and Yaote Chang. Design of decentralised adaptive sliding mode controllers for large-scale systems with mismatched perturbations. International Journal of Control, 81(10):1507-1518, 2008.

[3] C. Edwards and S. K. Spurgeon. Sliding mode control: Theory and applications. Taylor \& Francis, London, 1998.

[4] Giuseppe Fusco and Mario Russo. Design of decentralized robust controller for voltage regulation and stabilization of multimachine power systems. International Journal of Control, Automation and Systems, 11(2):277-285, 2013.

[5] Kou-Cheng Hsu. Decentralized variable-structure control design for uncertain large-scale systems with series nonlinearities. International Journal of Control, 68(6):1231-1240, 1997.

[6] L. Jiang, Q.H. Wu, and J.Y. Wen. Decentralized nonlinear adaptive control for multimachine power systems via high-gain perturbation observer. Circuits and Systems I: Regular Papers, IEEE Transactions on, 51(10):2052-2059, Oct 2004.

[7] Zhong-Ping Jiang. Recent developments in decentralized nonlinear control. In Control, Automation, Robotics and Vision Conference, 2004. ICARCV 2004 8th, volume 1, pages 326-331, Kunming, China, 2004. IEEE.

[8] B Labibi and SM Mahdi Alavi. Inversion-free decentralised quantitative feedback design of large-scale systems. International Journal of Systems Science, pages 1-11, 2014.

[9] Yang Mi, Yang Fu, Chengshan Wang, and Peng Wang. Decentralized sliding mode load frequency control for multi-area power systems. Power Systems, IEEE Transactions on, 28(4):4301-4309, Nov 2013.

[10] Jianqiu Mu, Xing-Gang Yan, and Sarah K Spurgeon. Decentralised sliding mode control for a class of nonlinear interconnected systems. In American Control Conference (ACC), 2015, pages 5170-5175. IEEE, 2015.

[11] Yugang Niu, T Jia, J Huang, and J Liu. Design of sliding mode control for neutral delay systems with perturbation in control channels. Optimal Control Applications and Methods, 33(3):363-374, 2012.

[12] Yugang Niu, J Lam, and X Wang. Sliding-mode control for uncertain neutral delay systems. Control Theory and Applications, IEE Proceedings-, 151(1):38-44, 2004.

[13] Dragoslav D Siljak. Decentralized control of complex systems. Courier Corporation, 2011.

[14] $\mathrm{H}$ Trinh and M Aldeen. Decentralised feedback controllers for uncertain interconnected dynamic systems. Control Theory and Applications, IEE Proceedings D, 140(6):429-434, 1993.

[15] Valery A Ugrinovskii, Ian R Petersen, Andrey V Savkin, and Elena Ya Ugrinovskaya. Decentralized state-feedback stabilization and robust control of uncertain large-scale systems with integrally constrained interconnections. Systems \& Control Letters, 40(2):107-119, 2000.

[16] Vadim Utkin, Jürgen Guldner, and Jingxin Shi. Sliding mode control in electro-mechanical systems, volume 34. CRC press, 2009.

[17] Xing-Gang Yan, Christopher Edwards, and Sarah K Spurgeon. Decentralised robust sliding mode control for a class of nonlinear interconnected systems by static output feedback. Automatica, 40(4):613-620, 2004.

[18] Xing-Gang Yan, Christopher Edwards, Sarah K Spurgeon, and JAM Bleijs. Decentralised sliding-mode control for multimachine power systems using only output information. Control Theory and Applications, IEE Proceedings-, 151(5):627-635, 2004.

[19] Xing-Gang Yan, James Lam, and Guangzhong Dai. Decentralized robust control for nonlinear similar large-scale systems. Computer \& Electrical Engineering, 25(3):169-179, 1999.

[20] Xing-Gang Yan, Sarah K Spurgeon, and Christopher Edwards. Decentralised robust sliding mode control for a class of nonlinear interconnected systems by static output feedback. Journal of Optimization Theory and Application, 119(3):597-614, 2003.

[21] Xing-Gang Yan, Sarah K Spurgeon, and Christopher Edwards. Dynamic sliding mode control for a class of systems with mismatched uncertainty. European Journal of Control, 11(1):1-10, 2005.

Fig. 2. Time response of control signal 